Research Article

\title{
Coriolis Force Sliding Mode Control Method for the Rotary Motion of the Central Rigid Body-Flexible Cantilever Beam System in TBM
}

\author{
Chuanlu Zhou, ${ }^{1}$ Long Qin ${ }^{D},{ }^{1,2}$ Ming Chen, ${ }^{1}$ and Jingxiang Zhang ${ }^{1}$ \\ ${ }^{1}$ China Railway 14th Bureau Group Mega Shield Construction Engineering Co., Ltd, Nan Jing 210000, China \\ ${ }^{2}$ School of Mechanical Engineering, Hangzhou Dianzi University, Hangzhou 310018, China \\ Correspondence should be addressed to Long Qin; wzw3049972460@hdu.edu.cn
}

Received 22 October 2021; Revised 15 November 2021; Accepted 18 November 2021; Published 23 December 2021

Academic Editor: Qinghua Zhang

Copyright (C) 2021 Chuanlu Zhou et al. This is an open access article distributed under the Creative Commons Attribution License, which permits unrestricted use, distribution, and reproduction in any medium, provided the original work is properly cited.

Beam slab structure is often encountered in a complex tunnel boring machine. Beam slab structure is subject to dynamic load, which is easy to cause fatigue damage and affect its service life. Therefore, it is necessary to control the vibration of this kind of beam slab structure. In this study, the central rigid body-flexible beam model is established for the rotating beam and plate rotating around the $y$-axis. Based on the Hamilton variational principle, the dynamic equation of the central rigid body-flexible beam system is established, and the dynamic model of the central rigid body-flexible beam system considering the influence of Coriolis force and centrifugal force is given. The vibration control of the central rigid body-flexible beam system is studied. The vibration mode of the rotating Euler Bernoulli beam is determined by using the elastic wave and vibration mode theory. The influence of the rotating motion on the beam vibration is analyzed, and the variable structure control law is designed to suppress the beam vibration. Numerical simulation results show that the control method can effectively suppress the first-order and second-order vibration of the beam and verify the effectiveness of the control strategy.

\section{Introduction}

With the development of shield technology, superlarge diameter tunnel boring machine (TBM) is frequently used in civil construction and production. However, various vibrations during operation and tunneling have caused damage to the components in the TBM and difficult maintenance, which has increased safety during construction. Risks delay in the construction period. TBM usually has large flexible attachments. These flexible attachments have the characteristics of large span, light weight, low rigidity, weak structural damping, and low rigidity of the connection between the plates $[1,2]$. During the operation of the TBM and the tunneling process, certain disturbance can easily arouse the vibration of the structure. Such vibration is not easy to be found in the complex underground environment, and the vibration may last for a long time [3-5]. This will not only directly affect the normal operation of the shield but also cause fatigue of such structures in the TBM, affect the service life of the structure, and even cause structural damage. Therefore, the attitude adjustment of the TBM and the suppression of the lateral vibration of the flexible attachment have extremely important engineering significance.

The precise dynamic modeling of the structural system is directly related to the motion control strategy and control effect of the system. At present, in the dynamic analysis and vibration control of mechanical arms and rotating machinery, when the flexible beam plate rotates as shown in Figure 1, the central rigid body-flexible beam model [6-8] is widely used. The effects of Coriolis force and centrifugal force are not considered in the state analysis, which will cause large errors in the calculation and analysis. Therefore, the study of active vibration control of rotating beams and plates and the establishment of a more accurate model that reflects the vibration state of the structure is an important way to optimize the control method. 


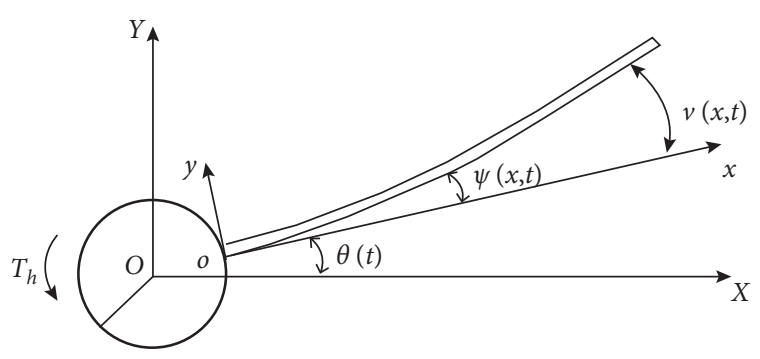

Figure 1: Model of the flexible hub-beam.

On the other hand, the coupling of rigid body rotation and flexural vibration of the flexible attachment will cause the system to be affected by centrifugal force and Coriolis force, which will cause the entire motion system to have time-varying and nonlinear characteristics. Look for a robust control strategy. It has become a hot issue in the vibration control of the central rigid body-flexible beam plate model [9-11]. The sliding mode variable structure control strategy is a robust control method, and it is currently widely used in active vibration control based on a central rigid body-flexible beam model [12-15].

Based on the Hamilton variational principle, this study adopts the Euler-Bernoulli beam theory to establish the dynamic equation of the central rigid body-flexible beam system for the rotating plate in which the rotating motion shown in Figure 1 occurs, and the centrifugal force and Coriolis force are considered in the modeling of the flexible attachment. Using elastic wave and vibration mode theory, the vibration mode of Euler-Bernoulli beam affected by Coriolis force is determined. The method of constant velocity approaching law is adopted to design the variable structure control law, and the control force is designed on the flexible attachment to suppress its lateral vibration.

\section{Dynamic Model System}

According to the movement state of the rotating plate, the model of the central rigid body-flexible beam system is shown in Figure 1. In the figure, $\mathrm{O}-\mathrm{XY}$ is a fixed inertial coordinate system, and $o-x y$ is a conjoined noninertial coordinate system. The radius of the central rigid body is $R_{h}$. The moment of inertia is $J_{h}$. The length, height, and mass density of the beam are $L, h$, and $\rho$. The load density of the control force is $f(x, t)$. The control torque is $T_{h}$. The angular displacement of the rigid body is $\theta_{t}$. The lateral displacement of the beam is $v(x, t)$. The Euler-Bernoulli beam theory is used in the dynamic analysis of the system, ignoring the influence of gravity and external disturbances.

During the rotational movement, the displacement of any point $(x, 0)$ on the beam relative to the fixed inertial coordinate system $O-\mathrm{XY}$ can be described as

$$
p=\left[-v \sin \theta-\left(R_{h}+x\right)(1-\cos \theta)\right] i+\left[v \cos \theta+\left(R_{h}+x\right) \sin \theta\right] j .
$$

When calculating the kinetic energy, the longitudinal deformation of the beam in motion is ignored. The total kinetic energy of the structure is the sum of the rotational kinetic energy of the central rigid body and the kinetic energy of the beam. Therefore, the total kinetic energy of the structural system is

$$
\begin{aligned}
E_{k} & =\frac{1}{2} \rho h \int_{0}^{L} \dot{p}^{2} \mathrm{~d} x+\frac{1}{2} J_{h} \dot{\theta}^{2} \\
& =\frac{1}{2} \rho h \int_{0}^{L}\left\{(-\dot{\theta} v)^{2}+\left[\dot{v}+\dot{\theta}\left(R_{h}+x\right)\right]^{2}\right\} \mathrm{d} x+\frac{1}{2} J_{h} \dot{\theta}^{2} .
\end{aligned}
$$

The bending strain energy of the structure is

$$
E_{p}^{(1)}=\frac{1}{2} D \int_{0}^{L} \psi^{\prime 2} \mathrm{~d} x,
$$

where $D=E I_{b}$ is the bending stiffness of the beam. $I_{b}$ is the moment of inertia of the beam. $\psi$ is the corner of the beam section, and ' $'$ ' is the derivative of space variable $x$.

When the flexible beam vibrates under the action of centrifugal force, it must overcome the centrifugal force to do work. When the beam vibrates, any cell $d m$ on the beam moves $\Delta x$ to the center $O$. For different microelement segments, $\Delta x$ is different. Therefore, the deformation energy generated by the centrifugal force on the beam is

$$
E_{p}^{(2)}=\int \Delta x \mathrm{~d} f_{c e}=\rho h \dot{\theta}^{2} \int_{0}^{L} \Delta x\left(R_{h}+x\right) \mathrm{d} x,
$$

where $d f_{c e}=\rho h \dot{\theta}^{2}\left(R_{h}+x\right) \mathrm{d} x$ is the centrifugal force received by the microelement, and $\Delta x$ is the amount of shortening of the structure in the axial direction caused by the lateral displacement.

$$
\Delta x=\frac{1}{2} \int_{0}^{x}\left(\frac{\partial v}{\partial \zeta}\right)^{2} \mathrm{~d} \zeta
$$

The deformation energy of the Coriolis force on the beam is

$$
E_{p}^{(3)}=\int v \mathrm{~d} f_{c 1}+\int \Delta x \mathrm{~d} f_{c 2}=2 \rho h \dot{\theta} \int_{0}^{L}(v \Delta \dot{x}+\dot{v} \Delta x) \mathrm{d} x,
$$

where $\mathrm{d} f_{c 1}=-2 \rho h \dot{\theta} \Delta \dot{x} \mathrm{~d} x$ is the Coriolis force that the infinite element receives in the tangential direction, and $d f_{c 2}=-2 \rho h \dot{\theta} \Delta \dot{v} \mathrm{~d} x$ is the Coriolis force received by the infinite element in the axial direction.

The work done by the external control force is

$$
W=T_{h} \theta+\int_{0}^{L} f(x, t) v(x, t) \mathrm{d} x+\int_{0}^{L}\left(R_{h}+x\right) f(x, t) \theta \mathrm{d} x,
$$

where $f(x, t)$ is the distributed load density of the beam, which can be expressed as

$$
f(x, t)=\sum_{i=1}^{k} F_{i}(t) \delta\left(x-x_{i}\right),
$$

where $F_{i}$ is the concentrated force on the beam where the abscissa is $x_{i}$.

The Hamilton principle of the system is 


$$
\int_{t_{0}}^{t_{f}} \delta\left(E_{k}-E_{p}+W\right) \mathrm{d} t=0
$$

Substituting (2)-(8) into (9), the dynamic equation of the central rigid body-flexible beam system is obtained as follows:

$$
\begin{aligned}
& \int_{0}^{L} \rho h\left[\theta v^{2}+\left(R_{h}+x\right)^{2} \ddot{\theta}-2\left(R_{h}+x\right) \ddot{\theta} \Delta x+\left(R_{h}+x\right) \ddot{v}-2 \ddot{v} \Delta x+2 \dot{\theta} v \dot{v}+2\left(R_{h}+x\right) \dot{\theta} \Delta \dot{x}+2 v \Delta \ddot{x}\right] \mathrm{d} x+J_{h} \ddot{\theta} \\
& \quad=T_{h}+\sum_{i=1}^{k} F_{i}(t)\left(R_{h}+x\right)_{i}, \\
& \quad \int_{0}^{L}\left(J_{b} \ddot{\psi}-D \psi^{\prime \prime}\right) \mathrm{d} x=0, \\
& \int_{0}^{L}\left\{\rho h\left[\left(R_{h}+x\right) \ddot{\theta}-2 \ddot{\theta} \Delta x+\ddot{v}-\dot{\theta}^{2} v-4 \dot{\theta} \Delta \dot{x}\right]+\frac{\partial}{\partial x}\left[v^{\prime} \int_{x}^{L} D_{0}(x, t) \mathrm{d} x\right]+D \psi^{\prime \prime \prime}-f(x, t)\right\} \mathrm{d} x=0,
\end{aligned}
$$

where

$$
D_{0}(x, t)=-\rho h\left[\left(R_{h}+x\right) \dot{\theta}^{2}+4 \dot{\theta} \dot{v}+2 \ddot{\theta} v\right] .
$$

The boundary conditions of the flexible beam are the lateral displacement of any point on the beam that can be written as

$$
\begin{aligned}
v(x, t) & =\sum_{i=1}^{n} \varphi_{i}(x) q_{i}(t) \\
& =\Phi \mathbf{q}
\end{aligned}
$$

where $\Phi=\left[\begin{array}{llll}\varphi_{1} & \varphi_{2} & \ldots & \varphi_{n}\end{array}\right], \quad q=\left[\begin{array}{llll}q_{1} & q_{2} & \ldots & q_{n}\end{array}\right]^{T}$, and $\varphi_{i}(x)$ and $q_{i}(t)$ are the $i^{\text {th }}$ modal function and modal coordinates corresponding to the lateral displacement of the flexible beam.

Therefore, the dynamic equation of the central rigid body-flexible beam system can be further written as

$$
\left[\begin{array}{ll}
M_{\theta \theta} & \mathbf{M}_{\theta q} \\
\mathbf{M}_{q \theta} & \mathbf{M}_{q q}
\end{array}\right]\left[\begin{array}{c}
\ddot{\theta} \\
\ddot{\mathbf{q}}
\end{array}\right]+\left[\begin{array}{cc}
0 & \mathbf{0} \\
\mathbf{0} & \mathbf{K}_{q q}
\end{array}\right]\left[\begin{array}{l}
\theta \\
q
\end{array}\right]+\left[\begin{array}{c}
Q_{\theta} \\
\mathbf{0}
\end{array}\right]=\left[\begin{array}{c}
T_{1} \\
\mathbf{T}_{2}
\end{array}\right],
$$

where $M_{\theta \theta} \in R^{1}$ is the total moment of inertia of the system; $\mathbf{M}_{q \theta} \in R^{n \times 1}, \mathbf{M}_{q \theta} \in R^{n \times 1}$ is the nonlinear inertial coupling between the rotational movement of the system and the lateral movement of the beam; $\mathbf{M}_{\mathrm{qq}} \in R^{n \times n}$ is the mass matrix of the flexible beam; $\mathbf{K}_{\mathrm{qq}} \in R^{n \times n}$ is the stiffness matrix; and $Q_{\theta} \in R^{1}$ is the inertial force term generated by the coupling of the rotation of the microelement and the lateral movement of the beam. Their specific expression forms are as follows:

$$
\begin{aligned}
& M_{\theta \theta}=J_{h}+J_{0}+q^{T} \mathbf{M}_{1} q-q^{T} \mathbf{M}_{2} q \\
& \mathbf{M}_{q \theta}^{T}=\mathbf{M}_{\theta q}=\mathbf{M}_{3} \\
& \mathbf{M}_{q q}=\mathbf{M}_{1}
\end{aligned}
$$

$$
\begin{aligned}
\mathbf{K}_{q q} & =-\dot{\theta}^{2}\left(\mathbf{M}_{1}-\mathbf{M}_{2}\right)+\mathbf{M}_{4} \\
\widetilde{\mathbf{Q}}_{\theta} & =2 \dot{\tilde{\theta}} \mathbf{q}^{T}\left(\mathbf{M}_{1}-\mathbf{M}_{2}\right) \dot{\mathbf{q}}
\end{aligned}
$$

$\widetilde{T}_{1}=\widetilde{T}_{h}+\sum_{i=1}^{k} \widetilde{F}_{i}(\widetilde{t})\left(\widetilde{R}_{h}+\widetilde{x}_{i}\right), \widetilde{T}_{2}=\sum_{i=1}^{k} \widetilde{F}_{i}(\widetilde{t}) \widetilde{\Phi}^{T}\left(\widetilde{x}_{i}\right)$

where $J_{0}=\int_{0}^{L} \rho \mathrm{h}\left(R_{h}+x\right)^{2} \mathrm{~d} x, \mathbf{M}_{1}=\int_{0}^{L} \rho h \Phi^{T} \Phi \mathrm{d} x, \mathbf{M}_{2}=\int_{0}^{L}$ $\rho h\left(R_{h}+x\right) \mathbf{D}_{1} \mathrm{~d} x, \quad \mathbf{M}_{3}=\int_{0}^{L} \rho h\left(R_{h}+x\right) \Phi \mathrm{d} x \quad \mathbf{M}_{4}=\int_{0}^{L} D \Phi^{\mathbf{T}}$ $\Phi^{\prime \prime \prime \prime} \mathrm{d} x, \quad \Delta x=1 / 2 \int_{0}^{x} v^{\prime 2}(x, t) \mathrm{d} x=1 / 2 q^{T} \mathbf{D}_{1} q, \quad \mathbf{D}_{1}=\int_{0}^{x} \Phi^{\prime T}$ $\Phi^{\prime} \mathrm{d} x$.

Define the dimensionless parameters $\tilde{t}=t \sqrt{E I / \rho h} / L^{2}$, $\widetilde{j}_{h}=j_{h} / \rho h L^{3},=\dot{\tilde{\theta}} \dot{\theta} L^{2} \sqrt{\rho h / E I}, \widetilde{T}_{1}=L T_{1} / E I, \widetilde{T}_{2}=L^{2} T_{2} / E I$, $\widetilde{x}=x / L, \widetilde{R}_{h}=R_{h} / L,=\widetilde{h} h / L$, and $\mathbf{q} / L=\widetilde{q}$. Then, the dynamic equation of the system in dimensionless form can be expressed as

$\left[\begin{array}{ll}\tilde{M}_{\theta \theta} & \tilde{\mathbf{M}}_{\theta \mathbf{q}} \\ \tilde{\mathbf{M}}_{\mathbf{q} \theta} & \widetilde{\mathbf{M}}_{\mathbf{q q}}\end{array}\right]\left[\begin{array}{c}\ddot{\tilde{\theta}} \\ \ddot{\tilde{q}}\end{array}\right]+\left[\begin{array}{cc}0 & 0 \\ 0 & \widetilde{\mathbf{K}}_{\mathbf{q q}}\end{array}\right]\left[\begin{array}{c}\tilde{\theta} \\ \widetilde{\mathbf{q}}\end{array}\right]+\left[\begin{array}{c}\tilde{Q}_{\theta} \\ \mathbf{0}\end{array}\right]=\left[\begin{array}{c}\widetilde{T}_{1} \\ \widetilde{\mathbf{T}}_{2}\end{array}\right]$.

The expression for the dimensionless parameter is

$\widetilde{M}_{\theta \theta}=\widetilde{J}_{h}+\widetilde{J}_{0}+\widetilde{\mathbf{q}}^{T} \widetilde{\mathbf{M}}_{1} \widetilde{\mathbf{q}}_{-} \widetilde{\mathbf{q}}^{T} \widetilde{\mathbf{M}}_{2} \widetilde{\mathbf{q}}$

$$
\begin{aligned}
\tilde{\mathbf{M}}_{q \theta}^{T} & =\tilde{\mathbf{M}}_{\theta q} \\
& =\tilde{\mathbf{M}}_{3},
\end{aligned}
$$

$\tilde{\mathbf{M}}_{q q}=\tilde{\mathbf{M}}_{1}$,

$\tilde{\mathbf{K}}_{q q}=-\dot{\tilde{\theta}}^{2}\left(\tilde{\mathbf{M}}_{1}-\tilde{\mathbf{M}}_{2}\right)+\tilde{\mathbf{M}}_{4}$

$\widetilde{\mathbf{Q}}_{\theta}=2 \dot{\tilde{\theta}} \widetilde{\mathbf{q}}^{T}\left(\widetilde{\mathbf{M}}_{1}-\tilde{\mathbf{M}}_{2}\right) \dot{\tilde{q}}$,

$\widetilde{T}_{1}=\widetilde{T}_{h}+\sum_{i=1}^{k} \widetilde{F}_{i}(\widetilde{t})\left(\widetilde{R}_{h}+\widetilde{x}_{i}\right), \widetilde{\mathbf{T}}_{2}=\sum_{i=1}^{k} \widetilde{F}_{i}(\widetilde{t}) \widetilde{\boldsymbol{\Phi}}^{T}\left(\widetilde{x}_{i}\right)$ 
where $\widetilde{J}_{0}=\int_{0}^{1}\left(\widetilde{R}_{h}+\widetilde{x}\right)^{2} \mathrm{~d} \widetilde{x}, \widetilde{\mathbf{M}}_{1}=\int_{0}^{1} \widetilde{\Phi}^{T} \widetilde{\Phi} \mathrm{d} \widetilde{x}, \widetilde{\mathbf{M}}_{2}=\int_{0}^{1}\left(\widetilde{R}_{h}\right.$ $+\widetilde{x}) \widetilde{\mathbf{D}}_{1} \mathrm{~d} x, \quad \widetilde{\mathbf{M}}_{3}=\int_{0}^{1}\left(\widetilde{R}_{h}+\widetilde{x}\right) \widetilde{\Phi} \mathrm{d} \widetilde{x}, \quad \widetilde{\mathbf{M}}_{4}=\int_{0}^{1} \widetilde{\Phi}^{\prime} T \widetilde{\Phi} \mathrm{d} \widetilde{x}$, $\Delta \widetilde{x}=1 / 2 \widetilde{q}^{T} \widetilde{\mathbf{D}}_{1} \tilde{q}$, and $\widetilde{D}_{1}=\int_{0}^{\tilde{x}} \widetilde{\Phi}^{\prime T} \widetilde{\Phi}^{\prime} \mathrm{d} \tilde{x}$.

Let $\mathbf{Y}=\left[\begin{array}{ll}\widetilde{\theta} & \widetilde{\mathbf{q}}^{T}\end{array}\right]^{T}$; then, there is

$$
\left[\begin{array}{cc}
\widetilde{M}_{\theta \theta} & \widetilde{\mathbf{M}}_{\theta q} \\
\widetilde{\mathbf{M}}_{q \theta} & \widetilde{\mathbf{M}}_{q q}
\end{array}\right] \ddot{\mathbf{Y}}+2 \dot{\theta}\left[\begin{array}{cc}
0 & \widetilde{\mathbf{G}}_{\theta q} \\
\mathbf{0} & \mathbf{0}
\end{array}\right] \dot{\mathbf{Y}}+\left[\begin{array}{cc}
0 & \mathbf{0} \\
\mathbf{0} & \widetilde{\mathbf{K}}_{q q}
\end{array}\right] \mathbf{Y}=\left[\begin{array}{c}
\widetilde{T}_{1} \\
\widetilde{\mathbf{T}}_{2}
\end{array}\right] \text {, }
$$

where $\quad \widetilde{\mathbf{G}}_{\theta q}=\widetilde{\mathbf{q}}^{T}\left(\widetilde{\mathbf{M}}_{1}-\widetilde{\mathbf{M}}_{2}\right)$.Let $\quad \mathbf{X}=\left[\begin{array}{ll}\mathbf{Y}^{T} & \dot{\mathbf{Y}}^{T}\end{array}\right]^{T} \quad \tilde{\mathbf{M}}=$ $\left[\begin{array}{ll}\widetilde{M}_{\theta \theta} & \widetilde{\mathbf{M}}_{\theta q} \\ \widetilde{\mathbf{M}}_{q \theta} & \widetilde{\mathbf{M}}_{q q}\end{array}\right] \widetilde{\mathbf{G}}=\left[\begin{array}{cc}0 & \widetilde{\mathbf{G}}_{\theta q} \\ \mathbf{0} & \mathbf{0}\end{array}\right] \mathbf{K}=\left[\begin{array}{cc}0 & 0 \\ \mathbf{0} & \widetilde{\mathbf{K}}_{q q}\end{array}\right] \widetilde{\mathbf{F}}=$ $\left[\begin{array}{lllll}1 & \widetilde{R}_{h}+\widetilde{x}_{1} & \ldots & \widetilde{R}_{h}+\widetilde{x}_{k} \\ 0 & \widetilde{\Phi}^{T}\left(\widetilde{x}_{1}\right) & \ldots & \widetilde{\Phi}^{T}\left(\widetilde{x}_{k}\right)\end{array}\right]$.

Then, the above formula can be further expressed as

$$
\dot{\mathbf{X}}=\left[\begin{array}{cc}
\mathbf{0} & \mathbf{I} \\
-\widetilde{\mathbf{M}}^{-1} \widetilde{\mathbf{K}} & -2 \dot{\boldsymbol{\theta}} \widetilde{\mathbf{M}}^{-1} \widetilde{\mathbf{G}}
\end{array}\right] \mathbf{X}+\left[\begin{array}{c}
\mathbf{0} \\
\widetilde{\mathbf{M}}^{-1} \mathbf{U} \widetilde{F}
\end{array}\right],
$$

where $\widetilde{\mathbf{F}}$ is the actuator distribution matrix, $\mathbf{U}=\left[\begin{array}{lllll}\widetilde{T}_{h} & \widetilde{F}_{1} & \cdots & \widetilde{F}_{k}\end{array}\right]^{T}$.

\section{Modal Analysis of Central Rigid Body- Flexible Beam}

When solving the beam mode, ignore the axial shortening caused by the lateral displacement and the external force $f(x, t)$ of the system, and consider the uniform rotation stage of the spacecraft's attitude adjustment, that is, the case where $=\dot{\theta} \Omega$ is a constant. From (10b), the dynamic equation of Euler-Bernoulli beam considering the influence of Coriolis force is

$$
\rho h\left(-\Omega^{2} v+\ddot{v}\right)+D \psi^{\prime \prime \prime}=0 .
$$

Let $v(x, t)=\varphi(x) e^{i \omega t}$ bring in the above data to get

$$
D \frac{\partial^{4} \varphi}{\partial x^{4}}-\rho h\left(\omega^{2}+\Omega^{2}\right) \varphi=0 .
$$

According to the elastic wave propagation theory and (20), the spatial wave number of the mode function $\varphi(x)$ should satisfy the following equation:

$$
k^{4}-\frac{\rho h}{D}\left(\omega^{2}+\Omega^{2}\right)=0
$$

When the elastic wave number $k_{i}^{2} \geq 0(i=1,2)$, it means that there are two pairs of propagating waves in the structural beam, and its phase velocity is $c=\omega / k$; when the elastic wave number $k_{1}^{2}>0$ and $k_{2}^{2}<0$, it means that there are a pair of propagating waves and a pair of attenuating waves in the structural beam. Since the structural vibration can be regarded as the superposition of multiple reflections of various elastic wave modes, the propagating wave can form the overall vibration in the limited area of the structure, and the attenuated wave can form the localized vibration in the limited area of the structure.
Therefore, the $j^{\text {th }}$ order vector mode function $\varphi_{j}(x)$ in the structure can be expressed as

$$
\varphi_{j}(x)=a_{1} \cos k_{1} x+a_{2} \sin \left(k_{1} x\right)+a_{3} \cos \left(k_{2} x\right)+a_{4} \sin \left(k_{2} x\right) .
$$

The mode coefficient that satisfies the cantilever beam boundary condition is

$$
\begin{aligned}
& a_{2}=-\frac{\left[k_{1}^{2} \cos \left(k_{1} l\right)-k_{2}^{2} \cos \left(k_{2} l\right)\right]}{\left[k_{1}^{2} \sin \left(k_{1} l\right)-k_{1} k_{2} \sin \left(k_{2} l\right)\right]} a_{1}, \\
& a_{4}=\frac{\left[k_{1}^{2} \cos \left(k_{1} l\right)-k_{2}^{2} \cos \left(k_{2} l\right)\right]}{\left[k_{2} k_{1} \sin \left(k_{1} l\right)-k_{2}^{2} \sin \left(k_{2} l\right)\right]} a_{1},
\end{aligned}
$$

where $k_{i}$ is the wave number of beam elastic vibration, which is determined by (21).

Satisfy the boundary conditions at both ends of the cantilever beam to obtain the following equation:

$$
\begin{aligned}
& k_{1}^{4} \sin ^{2}\left(k_{1} l\right)+k_{2}^{4} \sin ^{2}\left(k_{2} l\right)-\left(k_{1}^{2}+k_{2}^{2}\right) \times k_{1} k_{2} \sin \left(k_{1} l\right)\left(k_{2} l\right) \\
& \quad+k_{1}^{4} \cos ^{2}\left(k_{1} l\right)+k_{2}^{4} \cos ^{2}\left(k_{2} l\right)-2 k_{1}^{2} k_{2}^{2} \cos \left(k_{1} l\right) \cos \left(k_{2} l\right)=0 .
\end{aligned}
$$

Combining (21) and (24) together can determine the dispersion relation of vibration in the structure.

\section{Design of the Variable Structure Control Method}

The design of variable structure control mainly includes selecting the sliding mode and obtaining the control method. When the variable structure control strategy is adopted, the form of the switching function is as follows:

$$
\begin{aligned}
S & =\mathbf{C X} \\
& =\left[\begin{array}{llllll}
0 & 0 & 0 & 0 & 0 & 0 \\
0 & c_{1} & 0 & 0 & 1 & 0 \\
0 & 0 & c_{2} & 0 & 0 & 1
\end{array}\right] \mathbf{X},
\end{aligned}
$$

where $\mathbf{C}$ is the coefficient matrix, $\mathbf{X}=\left[\begin{array}{llllll}\theta & q_{1} & q_{2} & \dot{\theta} & \dot{q}_{1} & \dot{q}_{2}\end{array}\right]^{T}, S=0$, and these constitute the switching surface in variable structure control design. In the study of dynamics and control in this study, the first two vibration modes of the flexible attachment when rotating at a constant speed are considered.

Therefore, the specific form of the two switching surfaces is as follows:

$$
\begin{aligned}
& s_{1}=c_{1} q_{1}+\dot{q}_{1}, \\
& s_{2}=c_{2} q_{2}+\dot{q}_{2},
\end{aligned}
$$

where $q_{1}$ and $q_{2}$ are the first-order and second-order modal coordinates of the flexible attachment, respectively.

Use the following method of equal velocity approach

$$
\dot{s}_{i}=-P_{i} \operatorname{sgn}\left(s_{i}\right) \text {, }
$$



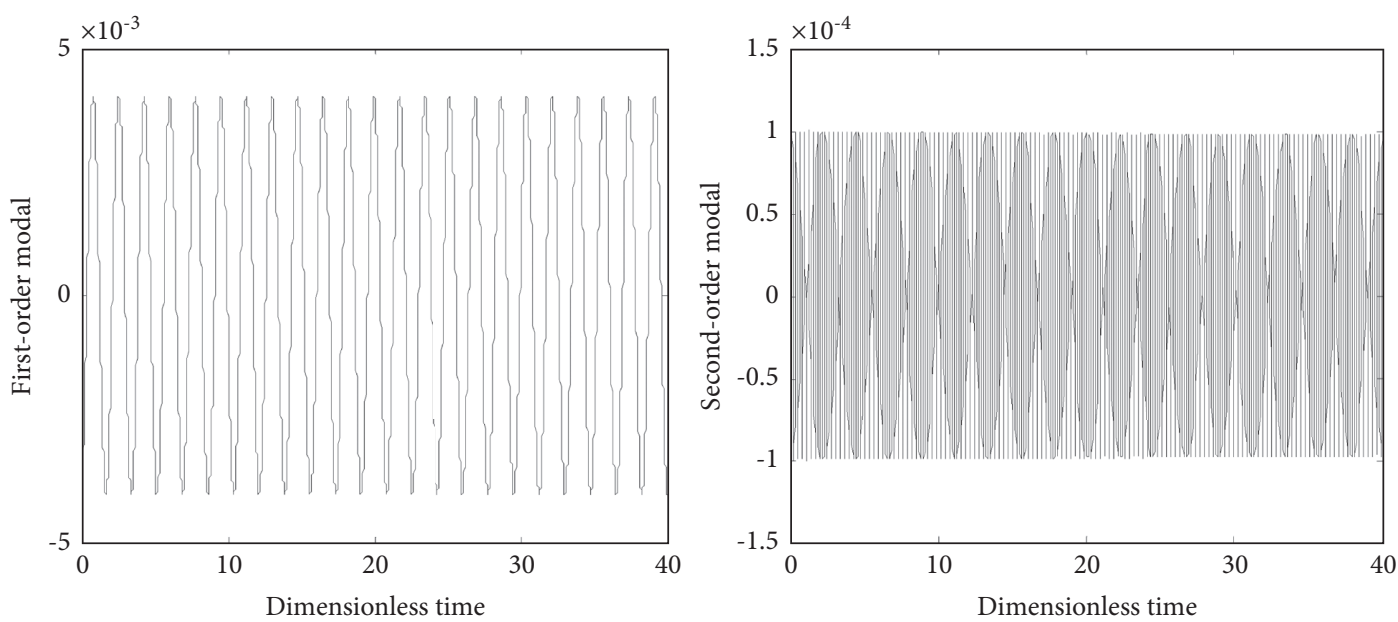

Figure 2: The response of the first two modes of the beam before variable structure control.
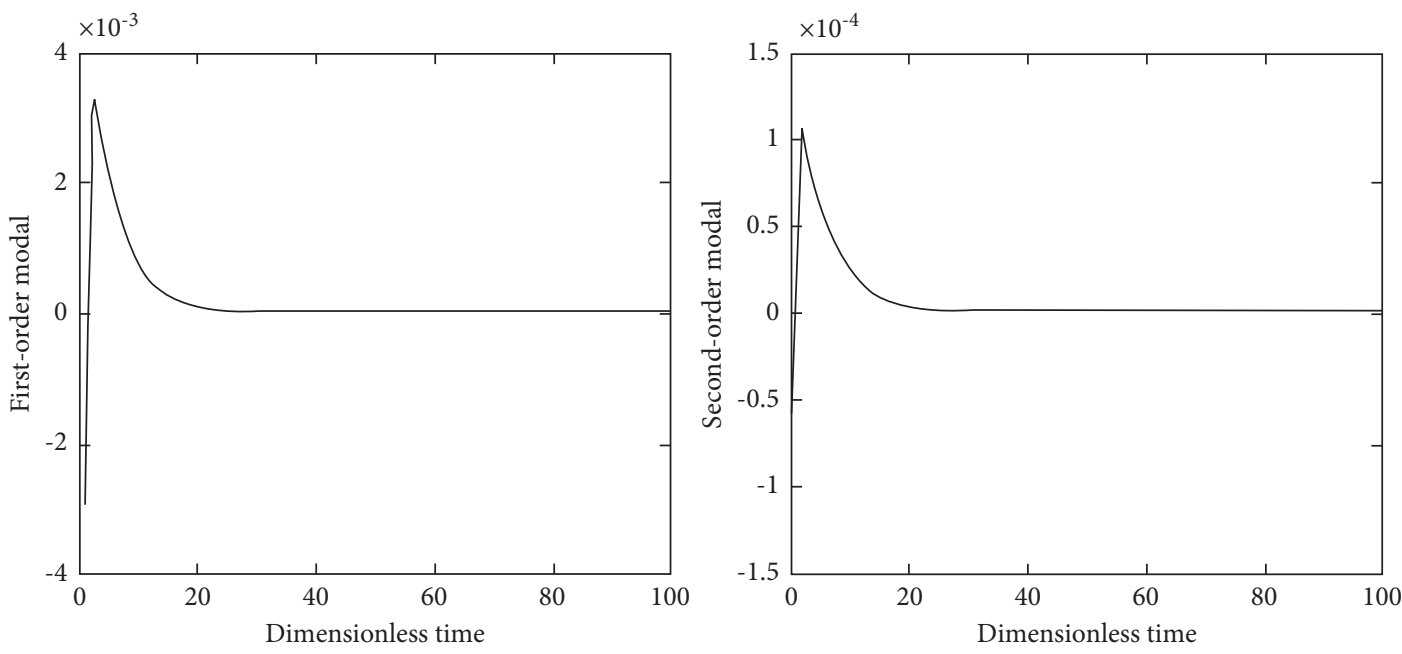

FIGURE 3: The response of the first two modes of the beam after variable structure control.

where $P_{i}>0, \operatorname{sgn}\left(s_{i}\right)=\left\{\begin{array}{cc}1 & s_{i}>0 \\ -1 & s_{i}<0\end{array}\right\}$. It is easy to verify that $\dot{s}_{i} s_{i}<0$ is established, and the reaching condition is satisfied.

Taking the derivation of (25) and considering (17) and (27), the equation satisfied by the variable structure control method is

$$
\mathbf{C}\left[\begin{array}{c}
\mathbf{0} \\
\tilde{\mathbf{M}}^{-1} \mathbf{U F}
\end{array}\right]=\left[\begin{array}{c}
0 \\
-P_{1} \operatorname{sgn}\left(s_{1}\right) \\
-P_{2} \operatorname{sgn}\left(s_{2}\right)
\end{array}\right]-\mathbf{C}\left[\begin{array}{cc}
\mathbf{0} & \mathbf{I} \\
-\tilde{\mathbf{M}}^{-1} \widetilde{\mathbf{K}} & -2 \dot{\boldsymbol{\theta}} \tilde{\mathbf{M}}^{-1} \widetilde{\mathbf{G}}
\end{array}\right] \mathbf{X} .
$$

Equation (17) becomes

$$
\mathbf{C} \dot{X}=\left[\begin{array}{c}
0 \\
-P_{1} \operatorname{sgn}\left(s_{1}\right) \\
-P_{2} \operatorname{sgn}\left(s_{2}\right)
\end{array}\right] \text {. }
$$

From equation (29), the solution at $P_{i}>0$ is stable, so the variable structure system is stable.

\section{Numerical Simulation}

In the calculation, the beam length $L$ is taken as the characteristic length. $v=0.30, \widetilde{J}_{h}=10 \widetilde{J}_{1}, \widetilde{R}_{h}=0.3$; consider the control when the rotation is a constant speed, and take parameters $P_{1}=\left|q_{1}\right|, P_{2}=\left|q_{2}\right|, c_{1}=0.2$, and $c_{2}=0.2$.

In this study, only the first two modes of the system are considered, but the control accuracy is good enough. The control force applied by the two actuators suppresses the vibration of the first-second mode of the flexible attachment, and the applied positions are $x_{1}=0.48 L$ and $x_{2}=0.96 L$, respectively.

Figure 2 shows the response of the first two-order transverse vibration mode coordinates of the flexible beam when the initial state of the system is $x=\left[\begin{array}{llllll}0 & -0.004 & -0.0001 & 1 & 0.002 & 0.0001\end{array}\right]$. It can be seen from the figure that before the variable structure control, the lateral vibration of the beam is severe.

Figure 3 shows the change rule of the coordinate of the first second-order transverse vibration mode of the flexible 

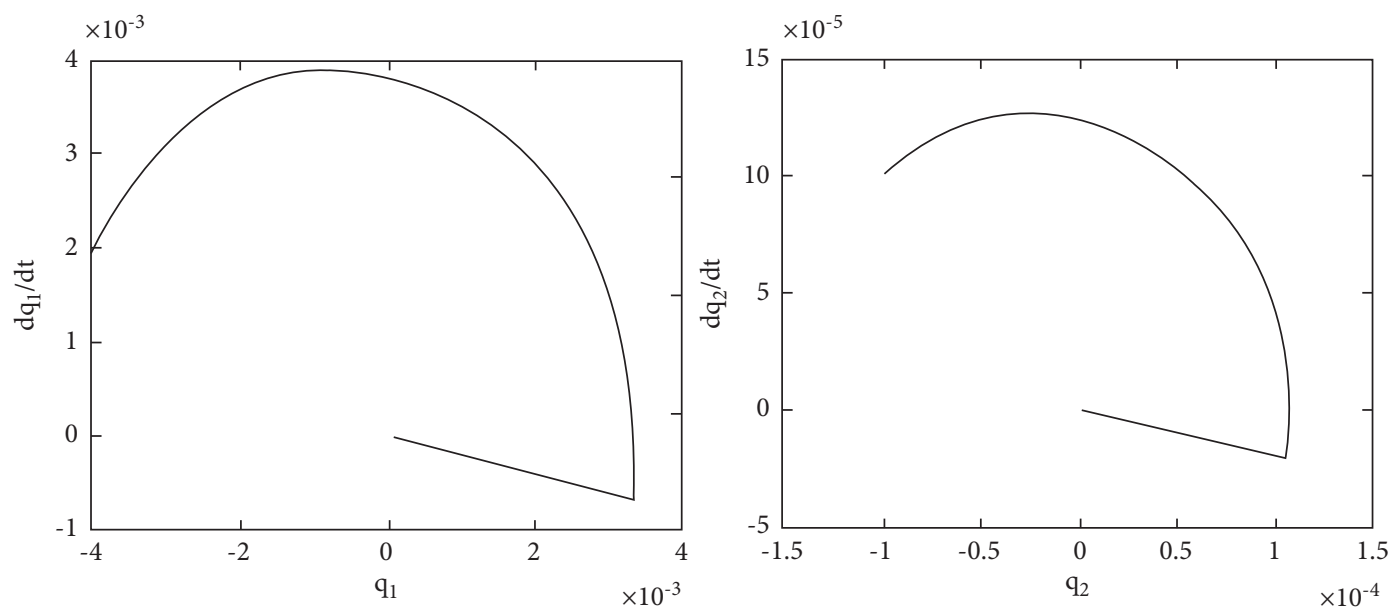

Figure 4: The response of the phase trajectory of the first two modes of the beam after variable structure control.
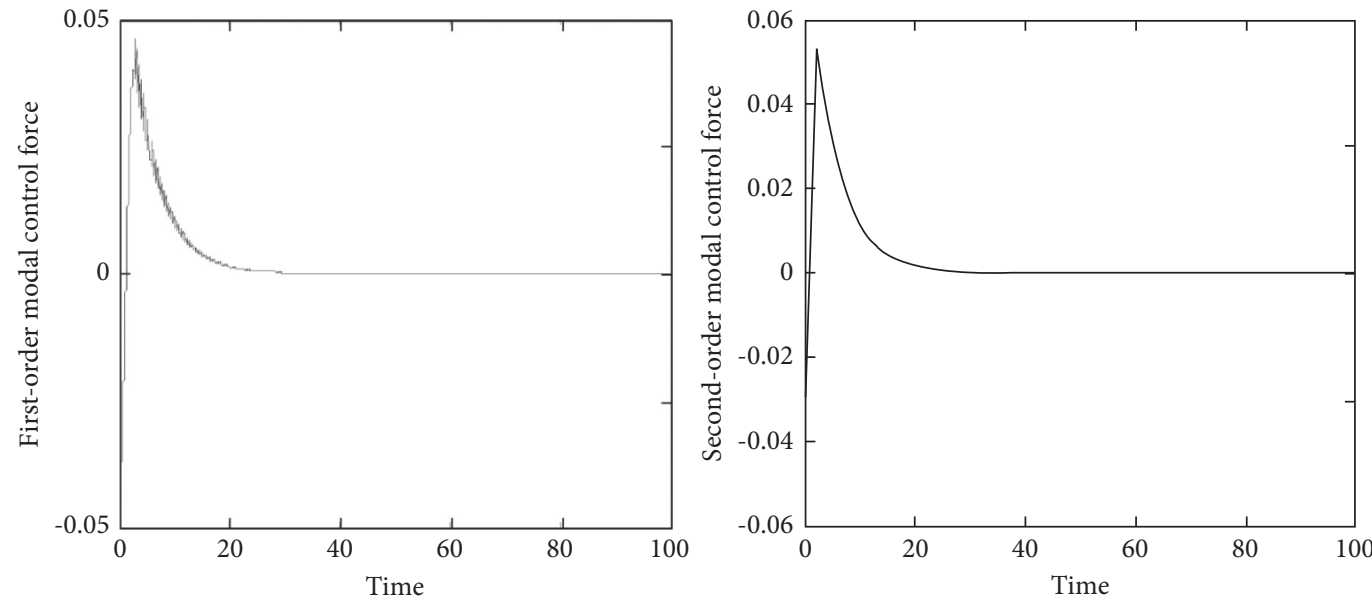

Figure 5: The time history of control inputs of the first two modes.

beam after variable structure control. It can be seen from the figure that the dimensionless time for the first stage to reach the desired position is about 17, and the dimensionless time for the second stage to reach the desired position is about 18 . The control effect is significant.

Figure 4 shows the change rule of the phase trajectory of the first two modes after the variable structure control. It can be clearly seen from the figure that the variable structure control makes the system enter the sliding mode and moves the modal coordinates along the sliding mode. Convergence is reached.

Figure 5 shows the changing law of the control force required by the system to reach the desired position. It can be seen from the figure that after the system reaches the desired position, the required control force tends to stabilize, which shows that the control strategy is effective.

\section{Conclusion}

Aiming at the vibration and Coriolis force during the operation and tunneling of the TBM, this study simulates the rotating beam plate rotating around the $Y$ axis as a central rigid body-flexible beam model for research. In the modeling process of the central rigid body-flexible beam system, the longitudinal shortening caused by the lateral displacement of the flexible beam is considered, and the influence of centrifugal force and Coriolis force is further improved, thereby, further improving the dynamic model. Based on the application of elastic wave and vibration mode theory, the vibration mode of the Euler-Bernoulli beam rotating at a constant speed is determined, and the influence of the rotation motion on the system is fully considered.

In this study, the innovation point is that the variable structure control strategy is applied to the active vibration control of the central rigid body-flexible beam model. The comparison of the simulation results shows that when the system vibrates, the system vibrates severely without active control. After adopting variable structure control, the stability of the system can be ensured, and the vibration of the flexible beam can be suppressed. The dynamic analysis and control method in this study can provide analysis methods and reference data for the dynamic analysis and control of the rotating beam structural components in the TBM. 


\section{Data Availability}

Thee data generated or analyzed during this research are included in this paper.

\section{Conflicts of Interest}

The authors declare that they have no conflicts of interest.

\section{Acknowledgments}

This work was supported by the National Natural Science Foundation of China (51875146).

\section{References}

[1] Z. Yang, Z. Sun, K. Fang, J. Yusheng, and B. Zhiqiang, "Cutting tool wear model for tunnel boring machine tunneling in heterogeneous grounds," Bulletin of Engineering Geology and the Environment, vol. 80, no. 7, pp. 5709-5723, 2021.

[2] Z. H. Zhang, M. Aqeel, C. Li, and F. Sun, "Theoretical prediction of wear of disc cutters in tunnel boring machine and its application," Journal of Rock Mechanics and Geotechnical Engineering, vol. 11, no. 1, pp. 111-120, 2019.

[3] Y. Shi, Y. Xia, Q. Tan, and Y. Zhang, "Distribution of contact loads in crushed zone between tunnel boring machine disc cutter and rock," Journal of Central South University, vol. 26, no. 9, pp. 2393-2403, 2019.

[4] J. P. Ou, Structural Vibration Control: Active, Semi-active and Intelligent Control, Science Press, Beijing, China, (in Chinese), 2003.

[5] Z. Qiu, M. Shi, B. Wang, and Z. Xie, "Genetic algorithm based active vibration control for a moving flexible smart beam driven by a pneumatic rod cylinder," Journal of Sound and Vibration, vol. 331, no. 10, pp. 2233-2256, 2012.

[6] H. Diken, "Vibration control of a rotating Euler-Bernoulli beam," Journal of Sound and Vibration, vol. 232, no. 3, pp. 541-551, 2000.

[7] J. B. Yang, L. J. Jiang, and D. C. Chen, "Dynamic modeling and control of a rotating Euler Bernoulli beam," Journal of Sound and Vibration, vol. 274, no. 3-5, pp. 863-875, 2004.

[8] N. Shafiei, M. Kazemi, and M. Ghadiri, "Comparison of modeling of the rotating tapered axially functionally graded Timoshenko and Euler-Bernoulli microbeams," Physica E: Low-Dimensional Systems and Nanostructures, vol. 83, pp. 74-87, 2016.

[9] I. Z. M. Darus and M. O. Tokhi, "Soft computing-based active vibration control of a flexible structure," Engineering Applications of Artificial Intelligence, vol. 18, no. 1, pp. 93-114, 2005.

[10] J. Lin and W.-Z. Liu, "Experimental evaluation of a piezoelectric vibration absorber using a simplified fuzzy controller in a cantilever beam," Journal of Sound and Vibration, vol. 296, no. 3, pp. 567-582, 2006.

[11] S. S. Ge, T. H. Lee, G. Zhu, and F. Hong, "Variable structure control of a distributed-parameter flexible beam," Journal of Robotic Systems, vol. 18, no. 1, pp. 17-27, 2001.

[12] Q. Hu and G. Ma, "Variable structure control and active vibration suppression of flexible spacecraft during attitude maneuver," Aerospace Science and Technology, vol. 9, no. 4, pp. 307-317, 2005.

[13] S. Choi, C. Cheong, and H. Shin, "Sliding mode control of vibration in a single-link flexible arm with parameter variations," Journal of Sound and Vibration, vol. 179, no. 5, pp. 737-748, 1995.

[14] T. Chen, C. Hu, and W. H. Huang, "Variable structure control and vibration suppression of spacecraft during attitude maneuver," Journal of Astronautics, vol. 28, no. 5, pp. 1199-1204, 2007.

[15] H. Bang and C. Ha, "Flexible spacecraft attitude maneuver by application of sliding mode control," Acta Astronautica, vol. 57, no. 11, pp. 841-850, 2005. 